\title{
Relaxation oscillations during the laser-induced spin state transition of a $\left[\mathrm{Fe}(\mathrm{PM}-\mathrm{BiA})_{2}(\mathrm{NCS})_{2}\right]$ complex
}

\author{
B. Viquerat, ${ }^{1,2}$ J. Degert, ${ }^{1,2}$ J. F. Létard, ${ }^{3}$ and E. Freysz ${ }^{1,2}$ \\ ${ }^{1}$ Université de Bordeaux, LOMA, UMR 5798, 351 Cours de la Libération, 33405 Talence Cedex, France \\ ${ }^{2}$ CNRS, LOMA, UMR 5798, 351 Cours de la Libération, 33405 Talence Cedex, France \\ ${ }^{3}$ CNRS, Université de Bordeaux, ICMCB, UPR 9048, 87 Avenue du Doc. A. Schweitzer, 33608 Pessac, France
}

(Received 28 August 2012; published 24 January 2013)

\begin{abstract}
We performed an optical pump-probe experiment to monitor the laser-induced spin state transition of [Fe(PM$\mathrm{BiA})_{2}(\mathrm{NCS})_{2}$ ] spin crossover microcrystallites. The sample is illuminated by a continuous-wave pump laser centered at $\lambda=976 \mathrm{~nm}$ that switches the compound from the low-spin to the high-spin state. When the pump laser is switched on or off, and above a given power threshold, the diffuse reflectivity of the sample increases or decreases, respectively, and presents characteristic damped oscillations that are not due to laser-induced excited spin state trapping. We propose a shell-core model that, due to the laser-induced heating and spin state transition of the microcrystallites, accounts very well for the reported observations.
\end{abstract}

DOI: 10.1103/PhysRevB.87.024303

PACS number(s): 05.70.Ln, 44.10.+i, 78.20.-e

Relaxation oscillations are nonlinear oscillations caused by a continuous increase of a constraint on a system followed by a sudden release of it. When stress becomes too high, the system abruptly gives way and a part of the energy stored is dissipated. Then the stress increases and the phenomenon occurs again. Relaxation oscillations are very common and have been recorded in many domains, such as biology, ${ }^{1}$ electrochemistry, ${ }^{2}$ lasers, ${ }^{3}$ as well as electronics. ${ }^{4}$ Hereafter, we demonstrate that they can also be observed during the laserinduced spin state phase transition in $\left[\mathrm{Fe}(\mathrm{PM}-\mathrm{BiA})_{2}(\mathrm{NCS})_{2}\right]$ microcrystallites. The latter material is an iron (II) spin crossover (SCO) compound in which the iron (II) ion exhibits an abrupt spin state transition (SST) from a low-spin (LS) state $(S=0)$ to a high-spin (HS) state $(S=2) .^{5}$ The SST is accompanied by a phase transition of the compound that often has a small but marked hysteresis loop. During the phase transition, many properties of the material are modified. In this complex, the LS to HS state transition is characterized by a large change of absorption in the visible and near-infrared (near-ir) spectral ranges. ${ }^{6}$ A very interesting aspect of SCO compounds is their ability to switch from LS to HS states by many different means. ${ }^{7}$ In addition to temperature, it has been shown that pressure ${ }^{8}$ as well as light $^{9,10}$ can drive the spin state transition. The latter effect is referred to in the literature as light-induced excited spin state trapping (LIESST),${ }^{10}$ the reverse LIESST effect, ${ }^{11}$ lightinduced thermal hysteresis (LITH), ${ }^{5,12}$ as well as light-induced optical hysteresis $(\mathrm{LIOH}) .{ }^{13}$ One very interesting aspect of these effects is that, at low temperature, the photoinduced HS state is metastable. This makes it possible to write and store optical information in such compounds. However, data storage is only possible at low temperature. Indeed, above an upper temperature $T$ (LIESST) that is typically below $100 \mathrm{~K}$, the photoinduced HS state relaxes toward the LS state. To circumvent this limitation, it is also possible to write optical information within the thermal hysteresis loop of the compound. ${ }^{14-16}$ Here again, as long as the temperature of the sample is kept within the hysteresis loop, the information can be permanently stored. We have studied the kinetics of photoswitching below and within the hysteresis loop in different SCO compounds. ${ }^{17,18}$ Excitation of the compound by a pulsed laser results in a heating of the sample and largely accounts for the recorded photoswitching. ${ }^{17-19}$ In fact, for most of the SCO compounds, such a laser excitation induces the heating of the sample that locally drives the SST. ${ }^{20-22}$ Hereafter, we wanted to record and understand the kinetics of this effect realized under quasicontinuous wave (QCW) laser excitation for a $\left[\mathrm{Fe}(\mathrm{PM}-\mathrm{BiA})_{2}(\mathrm{NCS})_{2}\right]$ compound. Under these conditions, we have recorded relaxation oscillations during the SST. We will show that these oscillations are associated with the SST of the compound that results from a laser-induced heating of the sample. We will also demonstrate that in this temperature range the LIESST or LIOH effects are negligible. Instead, we will propose a simple model that accounts very well for the observed phenomena. It makes it possible to evaluate the impacts of the laser excitation and the different sample parameters on the recorded phenomena.

The thermal hysteresis loop and the photoinduced metastable HS state of the studied [Fe(PM-BiA $\left.)_{2}(\mathrm{NCS})_{2}\right]$ compound recorded with a SQUID has already been published elsewhere: ${ }^{23}$ below $70 \mathrm{~K}$, the LS to HS state photoswitching can be induced by the LIESST effect, whereas, above $75 \mathrm{~K}$, the photoinduced HS state relaxes rapidly. In this compound, the SST is associated with a first-order phase transition that has a small but marked hysteresis loop of $\sim 8 \mathrm{~K}$ width around $T=170 \mathrm{~K}$. Figure 1 presents in more detail this thermal hysteresis loop recorded using a diffusive light-scattering setup. ${ }^{14}$ The latter setup restitutes the thermal hysteresis loop of this sample, which is made of an ensemble of crystals of a few tens of microns in size. In this experiment, the sample is placed in a cryostat and the diffusive reflectivity of the sample is measured using an unfocused and weak CW probe laser beam, a few $\mathrm{mW}$ in power, that is centered at $\lambda_{\text {probe }}=$ $532 \mathrm{~nm}$. The intensity of this probe beam is weak enough to discard any LIESST contribution. The diffusive light reflected by the sample is collected by a small focal lens $(\sim 10 \mathrm{~cm})$ and is focused on a photodiode in front of which is placed an interference filter centered at $532 \mathrm{~nm}$. As shown by Fig. 1, the change of reflectivity makes it possible to monitor the evolution of the HS fraction within the sample, $n_{\mathrm{HS}}$, and 


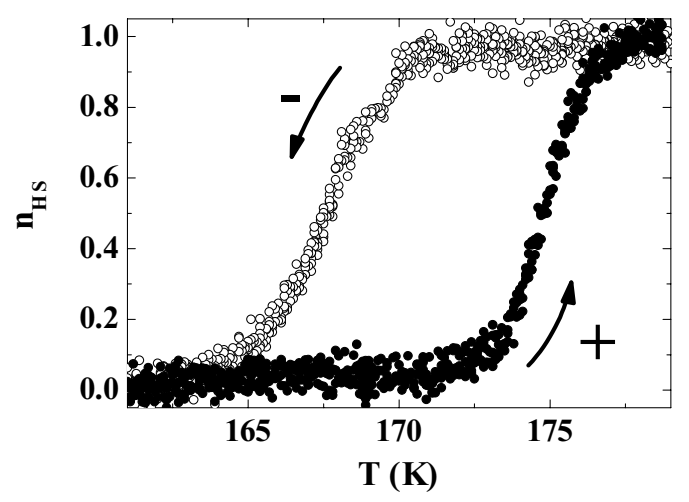

FIG. 1. Thermal hysteresis loop observed by increasing $(+)$ or decreasing $(-)$ the temperature, and recorded using a diffusive reflectance setup. $n_{\mathrm{HS}}$ is the fraction of complexes in the HS state, $n_{\mathrm{HS}}=1$ in the HS state (taken from Ref. 14).

to record the thermal hysteresis loop associated with the thermally activated SST. In the experiment reported hereafter, we used the same experimental setup. However, the sample is set in its LS state at $T=150 \mathrm{~K}$ and it is excited by a CW laser diode working at $\lambda_{\text {pump }}=976 \mathrm{~nm}$. The pump beam size on the sample is larger than the probe beam and is $\sim 5 \mathrm{~mm}$ in diameter.

The evolution of the sample reflectivity, $R$, recorded at $150 \mathrm{~K}$ when the pump laser was switched on for few tens of seconds and then switched off is shown in Fig. 2. In this figure, one can clearly notice the presence of a lagging time of 1 or $2 \mathrm{~s}$ between the switching of the laser and the reflectivity change of the sample and, also, the presence of oscillations of the reflectivity when the pump laser is either switched on or off. These damped oscillations last a few tens of seconds and then rapidly vanish. They only occur if the sample is in the LS state and when the pump beam is above a given power threshold. The threshold power decreases linearly as one increases the initial temperature of the sample. This trend is in good agreement with the pump power required to switch

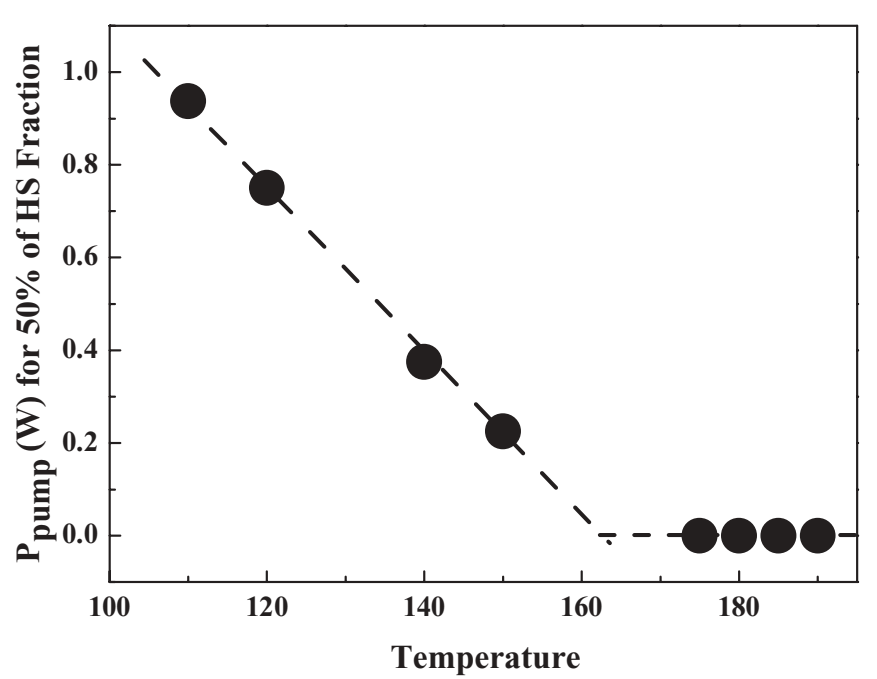

FIG. 3. Evolution of the pump power threshold vs the initial temperature of the sample. The pump threshold is defined as the pump intensity for which the reflectivity of the sample is equal to $50 \%$ of the reflectivity of the sample in the HS state. Above $175 \mathrm{~K}$ the sample is in the HS state.

$50 \%$ of the compounds from the LS state to the HS state versus the temperature of the sample (Fig. 3). Finally, the frequency of the oscillations depends on the pump intensity and the temperature of the sample (not shown here). The dark brown color of the sample is a good indication that it is partly absorbed in the near-ir spectral range. Hence for pump power as high as $1 \mathrm{~W}$, one cannot neglect the laser-induced heating of the sample. However, one may also wonder if the observed phenomena did not partly result from the LIESST effect. The answer to this question is in fact already given by our experiments. Indeed, Fig. 2(b) indicates that when the pump beam is switched off, i.e., in the absence of the LIESST effect, we also record these marked oscillations. Hence, the presence of these oscillations when the pump beam is off makes it possible to discard the LIESST effect as a possible cause. Hence we can conclude
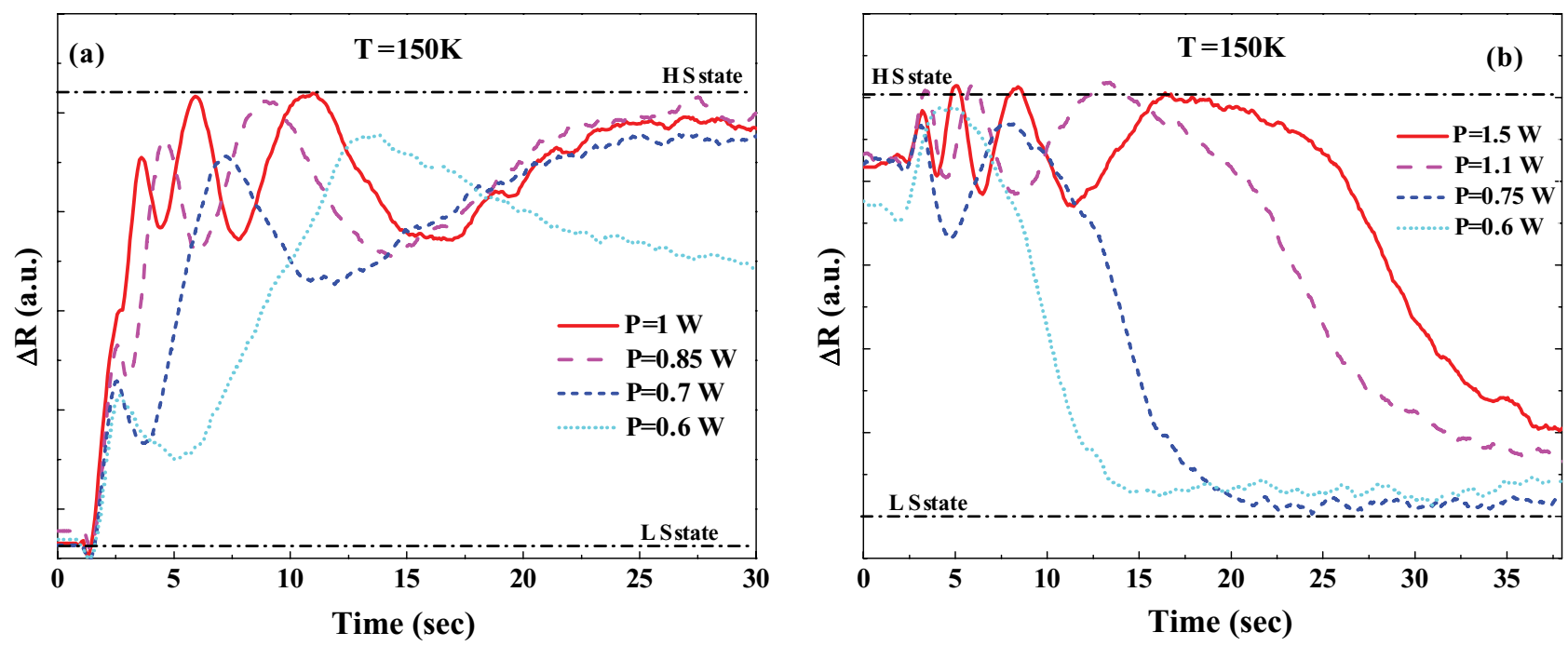

FIG. 2. (Color online) (a) Change of reflectivity when the pump laser is switched on for different laser powers. The HS fraction increases and oscillates before stabilizing to its final value. (b) Change of the reflectivity when the pump is switched off. 
that the laser-induced heating of the sample is the main effect responsible for the behavior recorded in Fig. 2.

Now let us try to understand what may happen to our sample set at $T=150 \mathrm{~K}$ when it is shined by a powerful pump laser beam. Since-as we previously mentioned-the sample absorbs part of the pump energy, its temperature has to increase. The temperature increase is proportional to the pump power. If the latter is high enough, the temperature of the sample may become higher than $T=173 \mathrm{~K}$. At this latter temperature the HS fraction $n_{\mathrm{HS}}$ starts to grow, and in agreement with Fig. 1, the sample reflectivity rapidly increases. As the sample reaches its equilibrium temperature, the reflectivity of the sample remains steady. At this steady point, the energy brought by the pump beam is balanced by the energy partly radiated by the particle and partly sunk by the cryostat. The higher the pump power, the higher the temperature increase of the sample, and the faster the switching from the LS to the HS state. Now if the pump power is switched off, the particles release most of their heat to the cryostat and cool down. When their temperature is below $168 \mathrm{~K}$, the sample reflectivity decreases rapidly (cf. Fig. 1). According to this naive picture, the reflectivity of the sample $R(t)$ should increase or decrease steadily versus time when the pump beam is switched on or off, respectively. Hence, and contrary to our experiment, $R(t)$ should exhibit no oscillations. In fact, the phenomenon is more complex. As we will show, a more realistic scenario should also account for the time it takes the sample to heat up and to switch between its HS and LS states. Moreover, the phase transition that is responsible for the reflectivity change of the sample is also accompanied by a change of other sample parameters, such as its specific heat or its absorption coefficient at the pump or probe wavelengths. The absorption of the sample is so high at the pump and probe wavelengths that one must account for the attenuation of the latter laser power within the sample. Thus, the pump attenuation induces a thermal gradient within each micrometric particle that is sensed by the probe beam. Hereafter, we propose a simple model that describes these phenomena. It basically accounts for the evolution of the temperature $T$ and $n_{\mathrm{HS}}$ within a micrometric particle. We will show that our model reflects our experimental observations and describes very well what we called "relaxation oscillations."

We will model our sample considering it is made of spherical crystallites having a diameter ranging from 10 to $100 \mu \mathrm{m}$. The temperature of the particles is fixed by the cryostat. The particles that absorb part of the pump energy are heated. However, because the compound is quite absorbent at the used wavelengths, most of the pump energy is absorbed at the surface of the particles within a layer of thickness $\lambda_{\text {pump }}$ with $\lambda_{\text {pump }}<1 \mu \mathrm{m} .{ }^{20}$ As already mentioned, the penetration length of the probe wavelength within the particles $\lambda_{\text {probe }}$ is also small. Hereafter, we will consider $\lambda_{\text {probe }} \sim \lambda_{\text {pump. }}$. To simplify the description of the evolution of the temperature within the particle when the laser is on, we will consider that the laser energy is released all along the surface of the particle within a layer of thickness $\lambda_{\text {pump }}$. We should therefore consider that the reflectivity changes are mainly due to the evolution of the fraction $n_{\mathrm{HS}}$ of these particles within $\lambda_{\text {pump }}$. This layer will be considered to be the "shell" of the particles. It can be easily shown that the evolution of the temperature within a homogeneous particle is as follows:

$$
\begin{aligned}
T(r, t)= & \frac{\alpha P\left(R_{\mathrm{tot}}^{2}-r^{2}\right)}{8 \rho C_{p} D_{\mathrm{th}}}+\frac{\alpha P R_{\mathrm{tot}}^{2}}{4 \pi^{2} \rho C_{p} D_{\mathrm{th}}} \\
& \times \sum_{n=1}^{\infty} \frac{(-1)^{n}}{n^{2}} \operatorname{sinc}\left(\frac{n \pi r}{R_{\mathrm{tot}}}\right) e^{-\frac{n^{2} \pi^{2} r}{R_{\mathrm{tot}}^{2}}},
\end{aligned}
$$

where $P, R_{\mathrm{tot}}, \alpha, \rho, C_{p}$, and $D_{\mathrm{th}}$ are, respectively, the laser power density, the radius, the absorption coefficient, the density, the heat capacity, and the thermal diffusivity of the particle. As indicated by Eq. (1), the temperature depends on many parameters. If, as occurs for iron (II) SCO compounds, the particle parameters depend on the spin state of the particle, then the "shell" temperature of the particle will depend on $n_{\mathrm{HS}}$ too. Indeed, iron (II) compounds in the LS state do have a broad absorption band centered at $850 \mathrm{~nm}$ that disappears in the HS state. This latter is broad enough, and has made it possible to induce the LIESST effect exciting the iron (II) complexes at $980 \mathrm{~nm} .{ }^{24}$ Let us also notice that, in this compound, the SST should also result in a jump of the thermal diffusivity, the density, and the heat capacity. This latter parameter is equal to 314 and $357 \mathrm{~J} \mathrm{Kg}^{-1} \mathrm{~K}^{-1}$ in the LS and HS states, respectively. ${ }^{5}$ The modulation of this ensemble of parameters results in an evolution of the temperature of the particle. For a homogeneous particle, the increase of the temperature of the particle is $\Delta T_{0}=\alpha P R_{\text {tot }}^{2} / 8 \rho C_{p} D_{\text {th }}$. To simplify our analysis, we will consider that the temperature of the inhomogeneous particle shell can be roughly approximated as follows:

$$
\Delta T_{0}(t) \approx \Delta T_{0, \mathrm{HS}}(t) n_{\mathrm{HS}}(t)+\Delta T_{0, \mathrm{BS}}(t)\left[1-n_{\mathrm{HS}}(t)\right],
$$

where $t$ is the time, $n_{\mathrm{HS}}$ is the HS fraction within the shell, and $\Delta T_{0, \mathrm{HS}}$ and $\Delta T_{0, \mathrm{LS}}$ are the shell temperatures for particles in the HS and LS states, respectively. Now let us explain the impact of Eq. (2) on the results presented in Fig. 2(a). At the beginning of the experiment, the particle is in the LS state and its temperature is fixed by the cryostat $\left(T_{0}=150 \mathrm{~K}\right)$. When the pump laser is switched on, the shell of the particles absorbs part of the pump energy and heats up. The heat slowly diffuses toward the core of the particles. This gives rise to a temperature gradient that evolves in time. As soon as the temperature of the shell is higher than the transition temperature, $n_{\mathrm{HS}}$ starts to grow. However, the growing time of $n_{\mathrm{HS}}$ within the shell is small compared to the time it takes the heat to diffuse toward the core of the particle. As $n_{\mathrm{HS}}$ increases, the net absorption of the particle diminishes. As a consequence, the absorption within the shell of the particle decreases. The shell experiences a drop in temperature that leads to a decrease of $n_{\mathrm{HS}}$. Then the sequence of phenomena we just described is repeated until the particle reaches its equilibrium temperature. To be more quantitative, we have simulated the evolution of $n_{\mathrm{HS}}(t)$ and $T(r, t)$ within a particle of the SCO compound. An example of our simulations is presented in Fig. 4 when $\alpha P=10 \mathrm{~mW} \mathrm{~cm}^{-2}$. It well reproduces our experimental data. Figure 4(a) presents the evolution of $n_{\mathrm{HS}}^{\mathrm{av}}(t)$, the averaged fraction of the complex in the HS state within the shell of the particle of $100 \mu \mathrm{m}$ diameter [i.e., $n_{\mathrm{HS}}^{\mathrm{av}}(t)=\left(1 / \lambda_{\text {probe }}\right) \int_{R_{\mathrm{tot}}}^{R_{\text {tot }}-\lambda_{\text {probe }}} n_{\mathrm{HS}}(r, t) d r$ ], as well as the temperature within the whole particle. In agreement with Fig. 1, the evolution of $n_{\mathrm{HS}}^{\mathrm{av}}(t)$ well reproduces the oscillations 

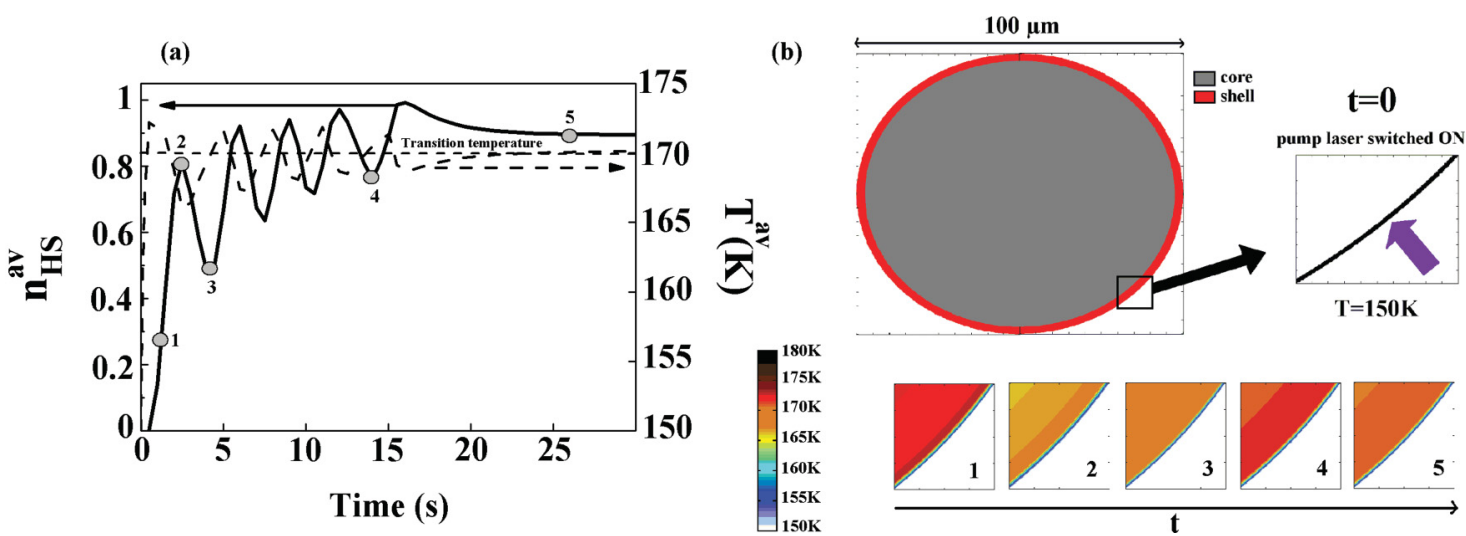

FIG. 4. (Color online) Theoretical evolution (a) of the averaged HS fraction, $n_{\mathrm{HS}}^{\mathrm{av}}(t)$, and averaged temperature of the particle shell thickness vs time upon $\mathrm{CW}$ laser excitation at $t=0$. As indicated by Fig. 1, the change of reflectivity shown in Fig. 2 reflects the evolution of the averaged $n_{\mathrm{HS}}$ within the particle shell thickness. (b) Temperature distribution within the particle at the times 1, 2, 3, 4, and 5 indicated in (a) when the $\mathrm{CW}$ exciting laser is switched on at $t=0$.

of $R(t)$. Figure 4(b) presents the evolution of the temperature gradient within the SCO particles. As expected, we have a sharp temperature gradient within the "shell." However, beyond the penetration depth of the pump beam $\lambda_{\text {pump }}$, one can almost neglect the pump absorption. Hence, it is mainly the heat diffusion that accounts for the increase of the temperature beyond $\lambda_{\text {pump. }}$. As time goes on, the temperature within the core and the "shell" of the particle increases. The following formula makes it possible to estimate the time $t_{h}$ it takes for the whole particle to increase its temperature by an amount of $\Delta T: \Delta T \sim 3 \alpha P t_{h} / R_{\text {tot }} \rho C_{p} .{ }^{17}$ Knowing the heat capacity, the density of our compound, ${ }^{5}$ and considering that the particle that is $100 \mu \mathrm{m}$ in diameter is absorbing a few percent of a laser beam having a power density of $1 \mathrm{~W} \mathrm{~cm}^{-2}$, we found that it takes $\sim 10 \mathrm{~s}$ for the particle to increase in temperature by $\sim 10 \mathrm{~K}$. It is interesting to notice that this rough estimate is in good agreement with our experimental data. The latter formula also makes it possible to evaluate the time it takes to heat up the "shell" of the particle. Replacing $R_{\text {tot }}$ by $\lambda_{\text {pump }}$, we found that it takes about $0.01 \mathrm{~s}$ to increase the temperature of the particle "shell" by $\sim 10 \mathrm{~K}$. This latter estimate is quite small compared to our simulations. However, it only takes into account the increase of temperature and not the time it takes for the HS domains to grow within the shell of the particle. Hence, the right time constant for the oscillation is in fact the time it takes for the HS domains to grow and/or to relax within the shell of the particle. It is this latter parameter that accounts for the lagging time. It is important to notice that the evolution of $n_{\mathrm{HS}}(t)$ is nonlinear ${ }^{24}$ and accounts for the increase of the characteristic oscillation time constant. We have neglected such dependence in our simulations. Figure 2(b) indicates that similar oscillations are recorded when the pump laser is switched off. To account for this observation, we propose a similar mechanism. When the pump laser is on for a few tens of seconds, the steady-state temperature is reached and $n_{\mathrm{HS}}=1$ in most of the particle. However, a large temperature gradient is kept within the shell of the particle [Fig. 4(b)]. Then when the laser is switched off, a large amount of energy is absorbed by the cryostat and the temperature of the particle has to decrease. In fact, the temperature drops very rapidly within the shell of the particle. Indeed, it is through the surface of the particle, in contact with the cryostat, that most of the heat is removed. However, it is important to keep in mind that the HS to LS transition is a first-order transition that has a specific latent heat. Hence, prior to the HS to LS transition, the system has to release its excess of energy $E_{\mathrm{ex}} \sim r^{3} \rho_{0} \mathrm{~T} \Delta \mathrm{C}_{p}$, where $\Delta \mathrm{C}_{p}$ is the specific-heat gap associated with the phase transition recorded at the temperature $T$ within a particle of radius $r$ and density $\rho_{0}$. Now, the time it takes for the whole particle to dissipate this excess of energy can be large compared to the time it takes for the SCO complexes within the shell of the particle to switch from the HS to the LS state. Hence, the molecules within the shell may be switched in the LS state before the core of the particle is cooled down. Once in the LS state, the shell of the particle has a lower heat capacity. Therefore, it senses the heat flowing from the bulk to the surface of the particle. This latter process results in an increase of the temperature of the shell that may drive it above $170 \mathrm{~K}$. At this latter temperature, the HS fraction starts to increase again and results in an increase of the reflectivity. This process will repeat until the temperature within the core of the particle becomes lower than $170 \mathrm{~K}$. We have simulated this process. The results of our numerical simulations are presented in Fig. 5, and they well reproduce our experimental data. It is interesting to notice that we could also account for the lagging time, which depends very slightly on the initial pump beam power and corresponds to the time it takes to remove the excess energy from the particle shell when the laser beam is switched off. Now looking at the oscillations presented in Fig. 2, it is interesting to examine the sample parameters that are necessary for their observation. Our model seems to indicate that the ratio $r_{a}=\lambda_{\text {pump }} / R_{\text {tot }}$ between the shell and the core of the particle is an important parameter. To address this point, we simulated the evolution of $n_{\mathrm{HS}}^{\mathrm{av}}(t)$ for different thicknesses of the particle shell [Fig. 6(a)]. As expected, the amplitude of the oscillations is reduced when $r_{a}$ gets closer to 0.1 , and they eventually vanish for larger values. We also wondered if this effect could be observed for an SCO compound that exhibits a large thermal hysteresis loop. Therefore, we ran our numerical model increasing the width $\Delta T$ of the hysteresis loop. Figure 6(b) shows our results: 

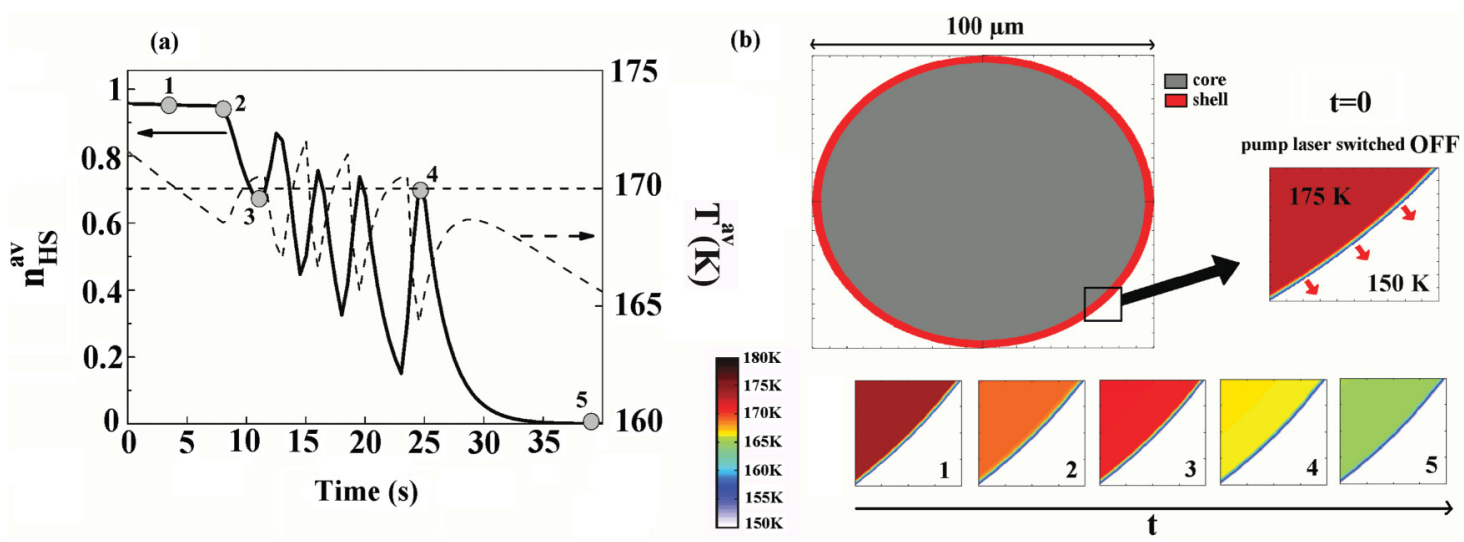

FIG. 5. (Color online) Theoretical evolution (a) of the averaged HS fraction, $n_{\mathrm{HS}}^{\mathrm{av}}(t)$, and averaged temperature of the particle shell thickness vs time after the CW laser excitation has been switched off at $t=0$. One can clearly notice the presence of a lagging time during which the temperature of the particle shell decreases while the reflectivity of the particle remains constant. (b) Temperature distribution within the particle at the times 1,2, 3, and 4 indicated in (a) when the CW exciting laser is switched off at $t=0$.

when the hysteresis loop gets wider, the oscillations lessen and eventually disappear. This indicates that the relaxation oscillations may be more difficult to show for particles making up a compound that has a larger thermal hysteresis loop. Finally, we have also simulated the evolution of the $n_{\mathrm{HS}}$ fraction for a compound without a thermal hysteresis loop. This indicates that the phenomena can also be recorded. In the latter case, the key parameter is the temperature range in which the compound switches from the LS to the HS state: the smaller this value is, the larger are the relaxation oscillations. In other words the relaxations oscillations will be difficult to record in SCO compounds that are either too cooperative or not cooperative enough. Finally, it is important to notice that the method we used to describe the phenomena is a first-order approximation. For instance, it cannot account for the slight increase of the oscillation frequency that one can notice in Fig. 2. More sophisticated models should be able to describe this behavior in more detail. ${ }^{25-27}$

In conclusion, we have shown that relaxation oscillations can be recorded during laser-induced spin state photoswitching. We have shown that these "relaxation oscillations" can be recorded because, on the one hand, the transition occurs within a sharp temperature range, and on the other hand, the pump beam is strongly absorbed within a thickness that is small compared to the radius of the particle. It is important to notice that such oscillations cannot be recorded using conventional techniques used to characterize SST such as SQUID or $\mathrm{x}$ rays, which usually indicate the evolution of the average HS fraction within the whole particle and are therefore less surface-sensitive. Finally, it is important to stress that this phenomenon should be easy to record during the laser-induced phase transition in a large variety of materials, and it is therefore not restricted to SCO compounds.

The authors would like to thank the GIS-Advanced Materials in Aquitaine (AMA), the ANR Agency (Ultimate-09BLAN-045198), and the Conseil Regional d'Aquitaine for supporting the COMMODORE program with the development of the COLA Platform (= Centre Optique et Laser d'Aquitaine) at the LOMA.
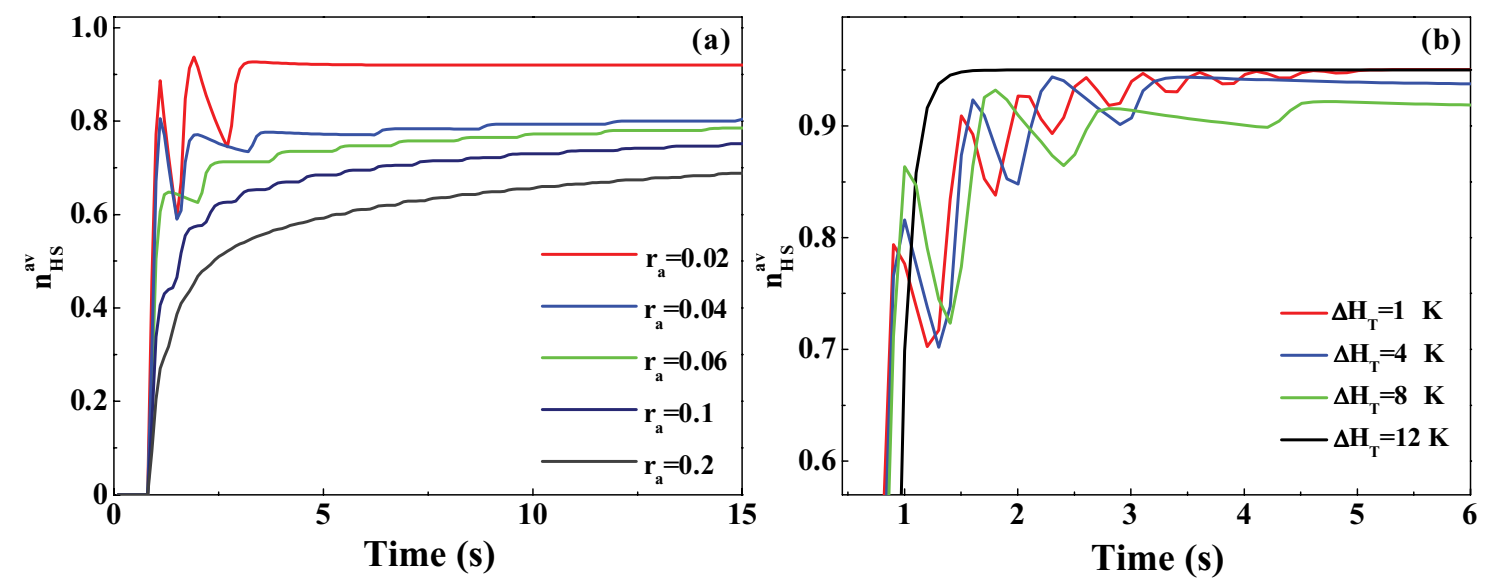

FIG. 6. (Color online) Theoretical evolution of $n_{\mathrm{HS}}^{\mathrm{av}}(t)$ vs (a) the thickness ratio between the shell and the core of the particle, and (b) the width of the thermal hysteresis loop. 


\section{APPENDIX}

We consider the heat propagation in a homogeneous sphere of radius $R_{\text {tot }}$ which is uniformly heated at its surface. The heat equation in that case is given by

$$
\frac{\partial T}{\partial t}=D \Delta T+P
$$

where $T$ is the temperature, $D$ is the thermal diffusivity of the material, and $P$ is the heat source.

Rewriting Eq. (A1) in spherical coordinates, we obtain

$$
\frac{\partial T}{\partial t}=D\left(\frac{2}{r} \frac{\partial T}{\partial r}+\frac{\partial^{2} T}{\partial r^{2}}\right)+P
$$

with the following boundary conditions:

$$
\begin{aligned}
\forall t, T\left(R_{\mathrm{tot}}, t\right) & =T_{1}, \\
\forall r, T(r, 0) & =T_{1},
\end{aligned}
$$

where $T_{1}$ is the temperature of the cryostat. Using the change of variables,

$$
G=r T+\frac{r^{3} P-r R_{\mathrm{tot}}^{2} P}{6 D}
$$

Eq. (A1) is written as

$$
\frac{\partial G}{\partial t}=D \frac{\partial^{2} G}{\partial r^{2}}
$$

with the following boundary condition:

$$
\begin{array}{r}
\forall t, G\left(R_{\mathrm{tot}}, t\right)=R_{\mathrm{tot}} T_{1}, \\
\forall r, G(r, 0)=\frac{r^{3} P-r R_{\mathrm{tot}}^{2} P}{6 D} .
\end{array}
$$

Then the Fourier series method yields

$$
G=\frac{2 P R_{\mathrm{tot}}^{3}}{D \pi^{3}} \sum_{n=1}^{\infty} \frac{(-1)^{n}}{n^{3}} \operatorname{sinc}\left(\frac{n \pi r}{R_{\mathrm{tot}}}\right) \exp \left(-\frac{n^{2} \pi^{2} D t}{R_{\mathrm{tot}}^{2}}\right) .
$$

In our case, $P$ is related to the pump laser and sample parameters. We can finally write the evolution of temperature within the spherical sample:

$$
\begin{aligned}
T(r, t)= & \frac{\alpha P\left(R_{\mathrm{tot}}^{2}-r^{2}\right)}{8 \rho C_{p} D}+\frac{\alpha P R_{\mathrm{tot}}^{2}}{4 \rho C_{p} D \pi^{2}} \\
& \times \sum_{n=1}^{\infty} \frac{(-1)^{n}}{n^{2}} \operatorname{sinc}\left(\frac{n \pi r}{R_{\mathrm{tot}}}\right) \exp \left(-\frac{n^{2} \pi^{2} D t}{R_{\mathrm{tot}}^{2}}\right) .
\end{aligned}
$$

${ }^{1}$ K. Kruse and F. Jülicher, Curr. Opinion Cell Biol. 17, 20 (2005).

${ }^{2}$ K. L. C. Hunt, P. M. Hunt, and J. Ross, Annu. Rev. Phys. Chem. 41, 409 (1990).

${ }^{3}$ A. E. Siegman, Lasers (University Science, Mill Valley, CA, 1986).

${ }^{4} \mathrm{P}$. Horowitz and W. Hill, The Art of Electronics (Cambridge University Press, Cambridge, UK, 1990).

${ }^{5}$ J. F. Létard, P. Guionneau, L. Rabardel, J. A. K. Howard, A. E. Goeta, D. Chasseau, and O. Kahn, Inorg. Chem. 37, 4432 (1998).

${ }^{6}$ A. Hauser, Coord. Chem. Rev. 11, 275 (1991).

${ }^{7}$ P. Gutlich and H. Goodwin, Top. Curr. Chem. 233, 1 (2004).

${ }^{8}$ A. Bousseksou, N. Negre, M. Goiran, L. Salmon, J. P. Tuchagues, M. L. Boillot, K. Boukheddaden, and J. F. Varret, Eur. Phys. J. B 13, 451 (2000).

${ }^{9}$ P. Gütlich, A. Hauser, and H. Spiering, Angew. Chem. Int. Ed. Engl. 33, 2024 (1994).

${ }^{10}$ S. Decurtins, P. Gütlich, C. P. Kohler, H. Spiering, and A. Hauser, Chem. Phys. Lett. 139, 1 (1984).

${ }^{11}$ A. Hauser, Chem. Phys. Lett. 124, 543 (1986).

${ }^{12}$ A. Hauser, R. Hinek, H. Spiering, and P. Gütlich, Chem. Eur. J. 2, 1435 (1996).

${ }^{13}$ A. Desaix, O. Roubeau, J. Jeftic, J. G. Haasnoot, K. Boukheddaden, E. Codjovi, J. Linares, M. Nogues, and F. Varret, Eur. Phys. J. B 6, 183 (1998).

${ }^{14}$ E. Freysz, S. Montant, S. Létard, and J.-F. Létard, Chem. Phys. Lett. 39, 318 (2004).

${ }^{15}$ S. Bonhommeau, J. J. McGarvey, J. A. Real, A. Zwick, G. Molnar, and A. Bousseksou, Angew. Chem. Int. Ed. 44, 4069 (2005).
${ }^{16}$ S. Cobo, D. Ostrovskii, S. Bonhommeau, L. Vendier, G. Molnár, L. Salmon, K. Tanaka, and A. Bousseksou, J. Am. Chem. Soc. 130, 9019 (2008).

${ }^{17}$ O. Fouché, J. Degert, G. Jonusauskas, N. Daro, J.-F. Létard, and E. Freysz, Phys. Chem. Chem. Phys. 12, 3044 (2010).

${ }^{18}$ O. Fouché, J. Degert, G. Jonusauskas, C. Baldé, C. Desplanches, J.-F. Létard, and E. Freysz, Chem. Phys. Lett. 469, 274 (2009).

${ }^{19}$ M. Lorenc, J. Hebert, N. Moisan, E. Trzop, M. Servol, M. BuronLeCointe, H. Cailleau, M. L. Boillot, E. Pontecorvo, M. Wulff, S. Koshihara, and E. Collet, Phys. Rev. Lett. 103, 028301 (2009).

${ }^{20} \mathrm{G}$. Galle, D. Deldique, J. Degert, E. Freysz, Th. Forestier, and J.-F. Létard, Appl. Phys. Lett. 96, 41907 (2010).

${ }^{21}$ G. Galle, J. Degert, C. Mauriac, C. Etrillard, J.-F. Letard, and E. Freysz, Chem. Phys. Lett. 500, 18 (2010).

${ }^{22}$ M. Lorenc, C. Balde, W. Kaszub, A. Tissot, N. Moisan, M. Servol, M. Buron-LeCointe, H. Cailleau, P. Chasle, P. Czarnecki, M. L. Boillot, and E. Collet, Phys. Rev. B 85, 054302 (2012).

${ }^{23}$ J.-F. Létard, G. Chastanet, O. Nguyen, S. Marcen, M. Marchivie, P. Guionneau, D. Chasseau, and P. Gütlich, Monatsh. Chem. 134, 165 (2003).

${ }^{24}$ P. Gütlich and A. Hauser, Coord. Chem. Rev. 97, 1 (1990).

${ }^{25}$ M. Nishino, K. Boukheddaden, Y. Konishi, and S. Miyashita, Phys. Rev. Lett. 98, 247203 (2007).

${ }^{26}$ L. Stoleriu, P. Chakraborty, A. Hauser, A. Stancu, and C. Enachescu, Phys. Rev. B 84, 134102 (2011).

${ }^{27}$ C. Enachescu, M. Nishino, S. Miyashita, L. Stoleriu, and A. Stancu, Phys. Rev. B 86, 054114 (2012). 